\title{
On Approximate Dynamic Inversion and Proportional-Integral Control
}

\author{
Justin Teo*, Jonathan P. How ${ }^{* *}$ \\ MIT Aerospace Controls Laboratory
}

\author{
Eugene Lavretsky ${ }^{\dagger}$ \\ Boeing Research \& Technology
}

\begin{abstract}
Approximate Dynamic Inversion (ADI) has been established as a method to control minimum-phase, nonaffinein-control systems. Previous results have shown that for singleinput nonaffine-in-control systems, every ADI controller admits a linear Proportional-Integral (PI) realization that is largely independent of the nonlinear function that defines the system. This paper extends these previous results in three ways. First, we present an extension of ADI that renders the closed loop error dynamics independent of the reference model dynamics. It is then shown that the equivalence between the ADI and PI controllers only holds for the time response when applied to the exact system. Finally, key robustness properties of the two control approaches are compared using linear system techniques. These results indicate that the PI realization is preferable when accurate knowledge of the nonlinear system dynamics is not available, and that the ADI realization would be preferred if time delays are the major limitations in the system.
\end{abstract}

\section{INTRODUCTION}

Dynamic inversion or feedback linearization is a popular control design method well suited for minimum-phase nonlinear systems [1] [2, Chapter 13]. It addresses the problem of controller design to transform a nonlinear system to a linear one by feedback. To overcome some limitations imposed by the requirements of exact linearization, approximate linearization has emerged as a viable alternative, where the problem is relaxed to enlarge the class of admissible controllers [3]. A notable departure from the approximate linearization literature is [4], where tracking control of nonaffine-in-control systems are considered.

An Approximate Dynamic Inversion (ADI) control law was proposed in [4] that drives a given minimum-phase nonaffine-in-control system towards a chosen stable reference model. The control signal was defined as a solution of "fast" dynamics, and Tikhonov's Theorem [2, Theorem 11.2, pp. $439-440$ ] in singular perturbation theory was used to show that the control signal approaches the exact dynamic inversion solution, and that the system states approach that of the reference model, when the controller dynamics are made sufficiently fast. A related technique in [5] uses high-gain filters to estimate some additive input uncertainties which in turn is used in the controller to cancel its effects.

In [6], we showed that for the single-input case, every ADI control law as formulated in [4] admits a linear Proportional-

\footnotetext{
* J. Teo is a Ph.D. candidate in the Department of Aeronautics \& Astronautics, Massachusetts Institute of Technology, Cambridge, MA 02139, USA. csteo@mit. edu

** J. P. How is a Professor in the MIT Department of Aeronautics \& Astronautics. jhow@mit.edu

$\dagger$ E. Lavretsky is a Boeing Senior Technical Fellow, The Boeing Company, Huntington Beach, CA 92647, USA. eugene. lavretskyaboeing.com
}

Integral (PI) model reference controller realization. The key characteristic of the equivalent PI controller is that it is largely independent of the system's nonlinearities, in contrast to the original ADI control law in [4]. However, when the controller have fast dynamics as required of the ADI method, the resulting PI controller is a high-gain controller with associated robustness problems [7]. This result can be seen as an extension of [8] to nonaffine-in-control systems and reference signals not necessarily approaching a constant limit, restricted to the state-feedback case.

In this paper, we extend the ADI method by decoupling the error dynamics specification from the reference model dynamics. This in essence decouples the "steady state" response specification from the transient response specification, when the reference model response is viewed as the "steady state" response. The equivalent PI controller for this extension can be similarly derived. The extension to multi-input systems is straightforward [9]. It will be shown that the equivalence between the ADI and PI controllers holds only for the time response when applied to the exact system. Finally, using linear system techniques, some robustness properties of the systems controlled by the ADI and PI controllers are established.

The rest of the paper is organized as follows. Section II presents the ADI extension and PI equivalent controller. Section III shows that this equivalence do not hold when the nominal system is perturbed. In the final section, by restricting consideration to minimum-phase Linear TimeInvariant (LTI) systems, some robustness properties of the two control laws are presented.

In the sequel, italicized symbols (eg. $x$ ) denote scalars, boldface lowercase letters (eg. $\mathbf{x}$ ) denote column vectors, and boldface uppercase letters (eg. A) denote matrices. Upright text subscripts (eg. $\mathrm{x}_{\mathrm{r}}$ with text subscript " $\mathrm{r}$ " to indicate state of reference model) are variable class indicators, and italicized subscript symbols (eg. $\mathbf{x}_{\rho}$ with subscript " $\rho$ " to indicate the $\rho$-th element of the vector $\mathbf{x}$ ) are variables for numeric quantities.

\section{Equivalence Between Approximate Dynamic INVERSION AND PI CONTROL}

\section{A. Approximate Dynamic Inversion for Single Input Systems}

Here, the ADI method [4] for single input systems is stated with a minor generalization, together with the main result. The proof in [4] applies with appropriate (trivial) substitutions, and will not be replicated here.

Consider an $n$-th order single-input nonaffine-in-control 
system of relative degree $\rho$, expressed in normal form

$$
\begin{aligned}
\dot{\mathbf{x}}(t)=\mathbf{f}(\mathbf{x}(t), \mathbf{z}(t), u(t)), & \mathbf{x}(0)=\mathbf{x}_{0}, \\
\dot{\mathbf{z}}(t)=\mathbf{g}(\mathbf{x}(t), \mathbf{z}(t), u(t)), & \mathbf{z}(0)=\mathbf{z}_{0},
\end{aligned}
$$

where $\mathbf{x}(t)=\left[x_{1}(t), x_{2}(t), \ldots, x_{\rho}(t)\right]^{\mathrm{T}} \in \mathbb{R}^{\rho}$,

$$
\mathbf{f}(\mathbf{x}(t), \mathbf{z}(t), u(t))=\left[\begin{array}{c}
x_{2}(t) \\
\vdots \\
x_{\rho}(t) \\
f(\mathbf{x}(t), \mathbf{z}(t), u(t))
\end{array}\right] \in \mathbb{R}^{\rho},
$$

for $(\mathbf{x}(t), \mathbf{z}(t), u(t)) \in D_{\mathbf{x}} \times D_{\mathbf{z}} \times D_{u}$, and the sets $D_{\mathbf{x}} \subset$ $\mathbb{R}^{\rho}, D_{\mathbf{z}} \subset \mathbb{R}^{n-\rho}$ and $D_{u} \subset \mathbb{R}$ are domains containing the origins. Here, $\left[\mathbf{x}^{\mathrm{T}}(t), \mathbf{z}^{\mathrm{T}}(t)\right]^{\mathrm{T}}$ denotes the state vector of the system, $u(t)$ is the control input, and $f: D_{\mathbf{x}} \times D_{\mathbf{z}} \times D_{u} \mapsto \mathbb{R}$, g: $D_{\mathbf{x}} \times D_{\mathbf{z}} \times D_{u} \mapsto \mathbb{R}^{n-\rho}$ are continuously differentiable functions of their arguments. Furthermore, assume that $\frac{\partial f}{\partial u}$ is bounded away from zero for $(\mathbf{x}(t), \mathbf{z}(t), u(t)) \in \Omega \subset$ $D_{\mathbf{x}} \times D_{\mathbf{z}} \times D_{u}$, where $\Omega$ is a compact set. That is, there exists $b_{0}>0$ such that $\left|\frac{\partial f}{\partial u}\right| \geq b_{0}$ for all $(\mathbf{x}(t), \mathbf{z}(t), u(t)) \in \Omega$. Note that $\left|\frac{\partial f}{\partial u}\right| \geq b_{0}>0$ implies $\operatorname{sign}\left(\frac{\partial f}{\partial u}\right) \in\{-1,+1\}$ is a constant. In addition, assume that the function $f$ cannot be inverted explicitly with respect to $u$.

It is desired for $\mathbf{x}(t)$ to track the states of a stable $\rho$-th order linear reference model described in the controllable canonical form

$$
\dot{\mathbf{x}}_{\mathrm{r}}(t)=\mathbf{A}_{\mathrm{r}} \mathbf{x}_{\mathrm{r}}(t)+\mathbf{B}_{\mathrm{r}} r(t), \quad \mathbf{x}_{\mathrm{r}}(0)=\mathbf{x}_{\mathrm{r} 0},
$$

where $\mathbf{x}_{\mathrm{r}}(t)=\left[x_{\mathrm{r} 1}(t), x_{\mathrm{r} 2}(t), \ldots, x_{\mathrm{r} \rho}(t)\right]^{\mathrm{T}} \in \mathbb{R}^{\rho}$, and the Hurwitz $\mathbf{A}_{\mathrm{r}}$ and column vector $\mathbf{B}_{\mathrm{r}}$ have the form

$$
\mathbf{A}_{\mathrm{r}}=\left[\begin{array}{cccc}
0 & 1 & \cdots & 0 \\
\vdots & \vdots & \ddots & \vdots \\
0 & 0 & \cdots & 1 \\
-a_{\mathrm{r} 0} & -a_{\mathrm{r} 1} & \cdots & -a_{\mathrm{r}(\rho-1)}
\end{array}\right], \quad \mathbf{B}_{\mathrm{r}}=\left[\begin{array}{c}
0 \\
\vdots \\
0 \\
b_{\mathrm{r}}
\end{array}\right] .
$$

Here, $r(t)$ is a continuously differentiable reference input signal, and $\mathbf{x}_{\mathrm{r}}(t)$ is the state of the reference model.

Let $\mathbf{e}(t)=\mathbf{x}(t)-\mathbf{x}_{\mathrm{r}}(t) \in \mathbb{R}^{\rho}$ be the tracking error signal, and let the desired stable error dynamics be specified by

$$
\dot{\mathbf{e}}(t)=\mathbf{A}_{\mathrm{e}} \mathbf{e}(t),
$$

where $\mathbf{A}_{\mathrm{e}}$ is Hurwitz and has identical structure as $\mathbf{A}_{\mathrm{r}}$, but with coefficients $a_{\mathrm{e} i}$ in place of $a_{\mathrm{r} i}$ for $i \in\{0,1, \ldots, \rho-1\}$.

Observe that in [4], $\mathbf{A}_{\mathrm{e}}$ was set equal to $\mathbf{A}_{\mathrm{r}}$, while in the above, an independent Hurwitz matrix $\mathbf{A}_{\mathrm{e}}$ can be specified. In typical applications, $\mathbf{A}_{\mathrm{r}}$ and $\mathbf{B}_{\mathrm{r}}$ can be used to specify the desired system response to excitation $r(t)$, and $\mathbf{A}_{\mathrm{e}}$ can be used to independently specify the desired error dynamics. That is, how quickly the system response approaches that of the reference model. Thus the preceding is a slight generalization of the ADI as formulated in [4].

The open loop (time-varying) error dynamics are then given by the system

$$
\begin{aligned}
& \dot{\mathbf{e}}(t)=\mathbf{f}\left(\mathbf{e}(t)+\mathbf{x}_{\mathrm{r}}(t), \mathbf{z}(t), u(t)\right)-\mathbf{A}_{\mathrm{r}} \mathbf{x}_{\mathrm{r}}(t)-\mathbf{B}_{\mathrm{r}} r(t), \\
& \dot{\mathbf{z}}(t)=\mathbf{g}\left(\mathbf{e}(t)+\mathbf{x}_{\mathrm{r}}(t), \mathbf{z}(t), u(t)\right),
\end{aligned}
$$

with initial conditions $\mathbf{e}(0)=\mathbf{e}_{0}, \mathbf{z}(0)=\mathbf{z}_{0}$. Define the selector vector $\mathbf{c}=[0, \ldots, 0,1]^{\mathrm{T}} \in \mathbb{R}^{\rho}$. The ideal dynamic inversion control is then found by solving the equation

$$
\mathbf{f}(\mathbf{x}(t), \mathbf{z}(t), u(t))-\mathbf{A}_{\mathrm{r}} \mathbf{x}_{\mathrm{r}}(t)-\mathbf{B}_{\mathrm{r}} r(t)=\mathbf{A}_{\mathrm{e}} \mathbf{e}(t),
$$

or equivalently,

$$
\begin{aligned}
f(\mathbf{x}(t), \mathbf{z}(t), u(t))-\mathbf{c}^{\mathrm{T}}\left(\mathbf{A}_{\mathrm{r}} \mathbf{x}_{\mathrm{r}}(t)+\right. & \left.\mathbf{B}_{\mathrm{r}} r(t)\right) \\
& =\mathbf{c}^{\mathrm{T}} \mathbf{A}_{\mathrm{e}} \mathbf{e}(t),
\end{aligned}
$$

resulting in the exponentially stable closed-loop tracking error dynamics (3). Since (5) cannot (in general) be solved explicitly for $u(t)$, the approximate dynamic inversion controller for the above formulation can be given in similar form to [4] as

$$
\epsilon \dot{u}(t)=-\operatorname{sign}\left(\frac{\partial f}{\partial u}\right) \tilde{f}(t, \mathbf{e}(t), \mathbf{z}(t), u(t)),
$$

where

$$
\begin{array}{r}
\tilde{f}(t, \mathbf{e}(t), \mathbf{z}(t), u(t))=f\left(\mathbf{e}(t)+\mathbf{x}_{\mathrm{r}}(t), \mathbf{z}(t), u(t)\right) \\
-\mathbf{c}^{\mathrm{T}}\left(\mathbf{A}_{\mathrm{r}} \mathbf{x}_{\mathrm{r}}(t)+\mathbf{B}_{\mathrm{r}} r(t)+\mathbf{A}_{\mathrm{e}} \mathbf{e}(t)\right),
\end{array}
$$

for some initial control $u(0)=u_{0}$. Here, $\epsilon$ is a positive controller design parameter, chosen sufficiently small to achieve closed-loop stability and approximate dynamic inversion. Observe that (6) relaxes the requirement for exact dynamic inversion while increasing the control in a direction to reduce the discrepancy (5) so as to approach the exact dynamic inversion solution.

Let $u=h(t, \mathbf{e}, \mathbf{z})$ be an isolated root of $\tilde{f}(t, \mathbf{e}, \mathbf{z}, u)=0$. In accordance with the theory of singular perturbations [2, Chapter 11], the reduced system for (4), (6) is

$$
\begin{array}{ll}
\dot{\mathbf{e}}(t)=\mathbf{A}_{\mathrm{e}} \mathbf{e}(t), & \mathbf{e}(0)=\mathbf{e}_{0}, \\
\dot{\mathbf{z}}(t)=\mathbf{g}\left(\mathbf{e}(t)+\mathbf{x}_{\mathrm{r}}(t), \mathbf{z}(t), h(t, \mathbf{e}(t), \mathbf{z}(t))\right), & \mathbf{z}(0)=\mathbf{z}_{0} .
\end{array}
$$

With $v=u-h(t, \mathbf{e}, \mathbf{z})$, and $\tau=t / \epsilon$, the boundary layer system is

$$
\frac{d v}{d \tau}=-\operatorname{sign}\left(\frac{\partial f}{\partial u}\right) \tilde{f}(t, \mathbf{e}, \mathbf{z}, v+h(t, \mathbf{e}, \mathbf{z})) .
$$

The main result of [4] for single-input systems, adapted for the generalization above, is stated below.

Theorem 1 (Hovakimyan et al. [4, Theorem 2]):

Assume that the following conditions hold for all $(t, \mathbf{e}, \mathbf{z}, u-h(t, \mathbf{e}, \mathbf{z}), \epsilon) \in[0, \infty) \times D_{\mathbf{e}, \mathbf{z}} \times D_{v} \times\left[0, \epsilon_{0}\right]$ for some domains $D_{\mathbf{e}, \mathbf{z}} \subset \mathbb{R}^{n}$ and $D_{v} \subset \mathbb{R}$, which contain the origins.

1) On any compact subset of $D_{\mathbf{e}, \mathbf{z}} \times D_{v}$, the functions $f$ and $\mathbf{g}$ and their first partial derivatives with respect to $(\mathbf{e}, \mathbf{z}, u)$, and the first partial derivative of $f$ with respect to $t$ are continuous and bounded, $h(t, \mathbf{e}, \mathbf{z})$ and $\frac{\partial f}{\partial u}(t, \mathbf{e}, \mathbf{z}, u)$ have bounded first derivatives with respect to their arguments, $\frac{\partial f}{\partial \mathbf{e}}(t, \mathbf{e}, \mathbf{z}, h(t, \mathbf{e}, \mathbf{z}))$ and $\frac{\partial f}{\partial \mathbf{z}}(t, \mathbf{e}, \mathbf{z}, h(t, \mathbf{e}, \mathbf{z}))$ are Lipschitz in $\mathbf{e}$ and $\mathbf{z}$, uniformly in $t$.

2) The origin is an exponentially stable equilibrium of the system

$$
\dot{\mathbf{z}}(t)=\mathbf{g}\left(\mathbf{x}_{\mathrm{r}}(t), \mathbf{z}(t), h(t, \mathbf{0}, \mathbf{z}(t))\right) .
$$


The mapping $(\mathbf{e}, \mathbf{z}) \mapsto \mathbf{g}\left(\mathbf{e}+\mathbf{x}_{\mathrm{r}}(t), \mathbf{z}, h(t, \mathbf{e}, \mathbf{z})\right)$ is continuously differentiable and Lipschitz in $(\mathbf{e}, \mathbf{z})$ uniformly in $t$.

3) $(t, \mathbf{e}, \mathbf{z}, v) \mapsto\left|\frac{\partial f}{\partial u}(t, \mathbf{e}, \mathbf{z}, v+h(t, \mathbf{e}, \mathbf{z}))\right|$ is bounded from below by some positive number for all $(t, \mathbf{e}, \mathbf{z}) \in$ $[0, \infty) \times D_{\mathbf{e}, \mathbf{z}}$.

Then the origin of (7) is exponentially stable. Moreover, let $\Omega_{v}$ be a compact subset of $R_{v}$, where $R_{v} \subset D_{v}$ denotes the region of attraction of the autonomous system

$$
\frac{d v}{d \tau}=-\operatorname{sign}\left(\frac{\partial f}{\partial u}\right) \tilde{f}\left(0, \mathbf{e}_{0}, \mathbf{z}_{0}, v+h\left(0, \mathbf{e}_{0}, \mathbf{z}_{0}\right)\right) .
$$

Then for each compact subset $\Omega_{\mathbf{e}, \mathbf{z}} \subset D_{\mathbf{e}, \mathbf{z}}$, there exists a positive constant $\epsilon_{*}$ and $T>0$ such that $\forall t \geq 0,\left(\mathbf{e}_{0}, \mathbf{z}_{0}\right) \in$ $\Omega_{\mathbf{e}, \mathbf{z}}, u_{0}-h\left(0, \mathbf{e}_{0}, \mathbf{z}_{0}\right) \in \Omega_{v}$, and $\forall \epsilon \in\left(0, \epsilon_{*}\right)$, system (1), (6) has a unique solution $\mathbf{x}_{\epsilon}(t)$ on $[0, \infty)$ and $\mathbf{x}_{\epsilon}(t)=\mathbf{x}_{\mathrm{r}}(t)+$ $O(\epsilon)$ holds uniformly for $t \in[T, \infty)$.

A proof of Theorem 1 is provided in [4]. In summary, Theorem 1 states that when regularity assumptions on the system dynamics are satisfied to ensure existence and uniqueness of solutions, and system (1) is minimum phase and controllable, the ADI control signal $u(t)$ approaches that of the exact dynamic inversion solution, and the system state $\mathbf{x}(t)$ approaches and maintains within $O(\epsilon)$ of the reference model state $\mathbf{x}_{\mathrm{r}}(t)$ for a sufficiently small $\epsilon$. See [4] for ways to verify the assumptions and further discussions.

\section{B. Equivalent PI Controller}

Here, we recall the main result of [6], [9], which extends trivially for the above ADI generalization.

Lemma 1: For every Approximate Dynamic Inversion controller (6) with $u(0)=u_{0}$, there exists a linear Proportional-Integral model reference controller realization

$$
\begin{aligned}
u(t)=-\frac{1}{\epsilon} \operatorname{sign}\left(\frac{\partial f}{\partial u}\right) & \left(\mathbf{c}^{\mathrm{T}} \mathbf{e}(t)\right. \\
& \left.-\int_{0}^{t} \mathbf{c}^{\mathrm{T}} \mathbf{A}_{\mathrm{e}} \mathbf{e}(\lambda) d \lambda-\tilde{u}_{0}\right)
\end{aligned}
$$

where $\tilde{u}_{0}=\mathbf{c}^{\mathrm{T}} \mathbf{e}(0)+\epsilon \operatorname{sign}\left(\frac{\partial f}{\partial u}\right) u_{0}$.

The proof is available in [9], using similar ideas from [6]. The key is to observe that the relations

$$
\begin{aligned}
f(\mathbf{x}(t), \mathbf{z}(t), u(t)) & =\mathbf{c}^{\mathrm{T}} \dot{\mathbf{x}}(t), \\
\mathbf{c}^{\mathrm{T}}\left(\mathbf{A}_{\mathrm{r}} \mathbf{x}_{\mathrm{r}}(t)+\mathbf{B}_{\mathrm{r}} r(t)\right) & =\mathbf{c}^{\mathrm{T}} \dot{\mathbf{x}}_{\mathrm{r}}(t),
\end{aligned}
$$

follow from (1) and (2) respectively.

It can be seen that the result (8) is a PI controller acting on the error between the system states and the states of the reference model. Furthermore, observe that when expressed in the error coordinates, e $(t)$, the PI controller is not explicitly dependent on $\mathbf{A}_{\mathrm{r}}$ that specifies the reference model dynamics, in contrast to the form in [6]. This characteristic is the result of introducing the independent matrix $\mathbf{A}_{\mathrm{e}}$ for error dynamics specification. From (8), it is apparent that the PI controller attempts to achieve (3), which is equivalent to achieving (5).

The significance of this result is threefold:
1) The PI controller allows a very simple exact realization of the ADI control law. Furthermore, no feedback of $\mathbf{z}(t)$ is required.

2) The PI controller is a linear realization of a (in general) nonlinear control law.

3) The PI controller realization is independent of the nonlinear function $f(\mathbf{x}(t), \mathbf{z}(t), u(t))$ in (1b), except for the sign of the control effectiveness, $\operatorname{sign}\left(\frac{\partial f}{\partial u}\right)$.

The existence of a linear realization of a nonlinear control law hinges critically on the structure of the underlying system, reference model, error dynamics and control law. The extension to multi-input nonaffine-in-control systems is straightforward [9]. In [6], [10], the equivalent PI controller is compared against the ADI variants in [11], [12] respectively. Because the PI controller is an exact realization, while the ADI variants in [11], [12] are approximate realizations, the PI realization achieves/exceeds the tracking performance of these ADI variants.

\section{Nonequivalence In Perturbed Systems}

Consider the scenario where the ADI control law is designed for a nominal system, but applied to a perturbed system. As stated above, a PI controller of the form (8) can be derived from the ADI control law (6). It is clear that when applied to the exact system (1), the PI controller (8) is equivalent to the ADI control law (6) in the sense that they produce identical time responses for the same excitation and initial conditions. As will be shown in Section IV, the equivalence only holds for the time response and not, in particular, to robustness properties. Equivalence in the time response also does not hold (in general) when these controllers are applied to a perturbed system. In particular, it is shown that this equivalence does not hold (in general) in the presence of:

1) disturbances at plant input/output,

2) perturbations of nonlinear function $f(\mathbf{x}(t), \mathbf{z}(t), u(t))$ in $(1 b)$, or

3) a single time delay at plant input/output.

The same conclusions hold for the multi-input case [9]. For notational convenience in the sequel, define

$$
\alpha=\operatorname{sign}\left(\frac{\partial f}{\partial u}\right) \in\{-1,1\} .
$$

\section{A. Disturbances at Plant Input/Output}

Let the system to be controlled be defined by (1) with

$$
u(t)=u_{\mathrm{c}}(t)+d_{\mathrm{i}}(t), \quad \mathbf{y}(t)=\left[\begin{array}{l}
\mathbf{x}(t) \\
\mathbf{z}(t)
\end{array}\right]+\left[\begin{array}{l}
\mathbf{d}_{\mathrm{xo}}(t) \\
\mathbf{d}_{\mathrm{zo}}(t)
\end{array}\right],
$$

where $u_{\mathrm{c}}(t) \in \mathbb{R}$ is the control signal, $d_{\mathrm{i}}(t) \in \mathbb{R}$ is the input disturbance, $\mathbf{y}(t) \in \mathbb{R}^{n}$ is the measurement, $\mathbf{d}_{\text {xо }}(t) \in \mathbb{R}^{\rho}$ and $\mathbf{d}_{\mathrm{zo}}(t) \in \mathbb{R}^{n-\rho}$ are output disturbances acting on $\mathbf{x}(t)$ and $\mathbf{z}(t)$ respectively. Define signal $h(t) \in \mathbb{R}$ as

$$
h(t)=-\mathbf{c}^{\mathrm{T}}\left(\mathbf{A}_{\mathrm{r}} \mathbf{x}_{\mathrm{r}}(t)+\mathbf{B}_{\mathrm{r}} r(t)+\mathbf{A}_{\mathrm{e}}\left(\mathbf{e}(t)+\mathbf{d}_{\mathrm{xo}}(t)\right)\right) .
$$


The ADI control law applied to the system with input and output disturbances defined by (11) is then given by

$$
\epsilon \dot{u}_{\mathrm{c}}(t)=-\alpha\left(f\left(\mathbf{x}(t)+\mathbf{d}_{\mathrm{xo}}(t), \mathbf{z}(t)+\mathbf{d}_{\mathrm{zo}}(t), u_{\mathrm{c}}(t)\right)+h(t)\right) .
$$

The PI controller applied to the same system is given by

$$
\begin{aligned}
u_{\mathrm{c}}(t)=-\frac{\alpha}{\epsilon} \mathbf{c}^{\mathrm{T}}(\mathbf{e}(t) & +\mathbf{d}_{\mathrm{xo}}(t) \\
& \left.-\mathbf{A}_{\mathrm{e}} \int_{0}^{t}\left(\mathbf{e}(\lambda)+\mathbf{d}_{\mathrm{xo}}(\lambda)\right) d \lambda\right),
\end{aligned}
$$

which, using (9) and (10), can be shown to be equivalent to the following control law

$$
\begin{aligned}
\epsilon \dot{u}_{\mathrm{c}}(t)=-\alpha(f(\mathbf{x}(t), \mathbf{z}(t), & \left.u_{\mathrm{c}}(t)+d_{\mathrm{i}}(t)\right) \\
& \left.+\mathbf{c}^{\mathrm{T}} \dot{\mathbf{d}}_{\mathrm{xo}}(t)+h(t)\right) .
\end{aligned}
$$

It can be seen that, in general, the two controllers given by (12) and (13) are equivalent only when $d_{\mathrm{i}}(t)=0$, $\mathbf{d}_{\mathrm{xo}}(t)=\mathbf{0}$, and $\mathbf{d}_{\mathrm{zo}}(t)=\mathbf{0}$ for all $t \geq 0$.

\section{B. Delay-free Perturbation of System}

If the system to be controlled is defined by (1), but with $f(\mathbf{x}(t), \mathbf{z}(t), u(t))$ perturbed to $f_{\mathrm{p}}(\mathbf{x}(t), \mathbf{z}(t), u(t))$, the ADI control law remains unaltered as in (6). With

$$
h(t)=-\mathbf{c}^{\mathrm{T}}\left(\mathbf{A}_{\mathrm{r}} \mathbf{x}_{\mathrm{r}}(t)+\mathbf{B}_{\mathrm{r}} r(t)+\mathbf{A}_{\mathrm{e}} \mathbf{e}(t)\right),
$$

the ADI control law can be rewritten as

$$
\epsilon \dot{u}(t)=-\alpha(f(\mathbf{x}(t), \mathbf{z}(t), u(t))+h(t)) .
$$

The PI controller, in contrast, can be shown to be equivalent to

$$
\epsilon \dot{u}(t)=-\alpha\left(f_{\mathrm{p}}(\mathbf{x}(t), \mathbf{z}(t), u(t))+h(t)\right) .
$$

Observe that (15) differs from (14) in the nonlinear function $f_{\mathrm{p}}(\mathbf{x}(t), \mathbf{z}(t), u(t))$. The independence of the PI controller from the nonlinear function renders it insensitive to delayfree perturbations of $f(\mathbf{x}(t), \mathbf{z}(t), u(t))$. It is clear that for the system described by (1) with $f(\mathbf{x}(t), \mathbf{z}(t), u(t))$ replaced by $f_{\mathrm{p}}(\mathbf{x}(t), \mathbf{z}(t), u(t))$, and controlled by (15), Theorem 1 applies unaltered. This implies that for all delay-free perturbations described by $f_{\mathrm{p}}(\mathbf{x}(t), \mathbf{z}(t), u(t))$, there exists a sufficiently small positive $\epsilon$ for which the PI controller stabilizes the system.

\section{A Single Time Delay at Plant Input/Output}

Here, we consider the case where there is a single delay present at the plant input/output. To simplify the exposition, we first state a fact which is a property of time-invariant systems. Let $T_{d}>0$ be the delay interval, and let signal $\mathbf{u}(t)$ be defined for $t \in\left[-T_{d}, 0\right]$. Let the input-delayed timeinvariant system with input $\mathbf{u}(t)$, output $\mathbf{y}(t)$, and state $\mathbf{x}(t)$, be defined by

$$
\begin{aligned}
& \dot{\mathbf{x}}(t)=\mathbf{f}\left(\mathbf{x}(t), \mathbf{u}\left(t-T_{d}\right)\right), \quad \mathbf{x}(0)=\mathbf{x}_{0}, \\
& \mathbf{y}(t)=\mathbf{g}\left(\mathbf{x}(t), \mathbf{u}\left(t-T_{d}\right)\right) .
\end{aligned}
$$

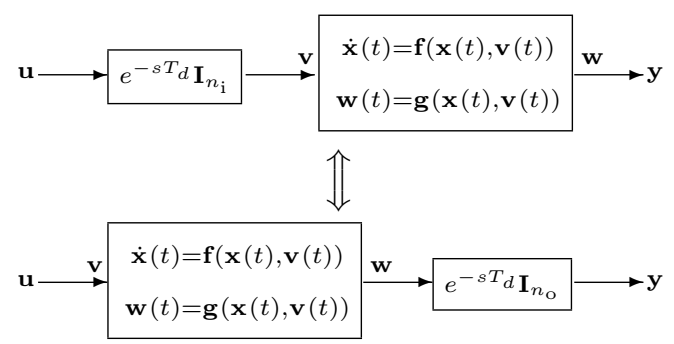

Fig. 1. A single delay at the input/output commutes with a time-invariant system.

Let the output-delayed time-invariant system with input $\mathbf{u}(t)$, output $\mathbf{y}(t)$, and state $\tilde{\mathbf{x}}(t)$, be defined by

$$
\begin{array}{ll}
\dot{\tilde{\mathbf{x}}}(t) & =\mathbf{f}(\tilde{\mathbf{x}}(t), \mathbf{u}(t)), \quad \tilde{\mathbf{x}}\left(-T_{d}\right)=\mathbf{x}_{0}, \\
\mathbf{y}(t) & =\mathbf{g}\left(\tilde{\mathbf{x}}\left(t-T_{d}\right), \mathbf{u}\left(t-T_{d}\right)\right) .
\end{array}
$$

The solution of systems (16) and (17) are well defined for $t \in\left[0, T_{d}\right]$. In particular, they have identical outputs during this interval.

Proposition 1: System (16) is equivalent to system (17) in the sense that both systems produce the same output when excited by the same input for all $t>0$.

Proof: Define $\tilde{\mathbf{x}}(t)=\mathbf{x}\left(t+T_{d}\right)$, and perform a change of the time variable.

Proposition 1 states that the delay operator commutes with time invariant systems at the input and output, with an appropriate change in input/output dimensions. This is illustrated in Fig. 1 schematically, where the delay operator is represented by $e^{-s T_{d}}$, and the input and output dimensions are $n_{\mathrm{i}}$ and $n_{\mathrm{o}}$ respectively. In Proposition 1, it is crucial that the nonlinear functions $\mathbf{f}$ and $\mathbf{g}$ are not explicit functions of the time variable, $t$.

With Proposition 1, it suffices to consider the case where the single delay appears at the input. The system to be controlled is therefore defined by (1) with

$$
u(t)=u_{\mathrm{c}}\left(t-T_{d}\right),
$$

where $T_{d}$ is the delay interval and $u_{\mathrm{c}}(t)$ is the control signal. With

$h(t)=-\mathbf{c}^{\mathrm{T}}\left(\mathbf{A}_{\mathrm{r}} \mathbf{x}_{\mathrm{r}}(t)+\mathbf{B}_{\mathrm{r}} r(t)+\mathbf{A}_{\mathrm{e}}\left(\mathbf{x}\left(t-T_{d}\right)-\mathbf{x}_{\mathrm{r}}(t)\right)\right)$,

the ADI control law takes the form

$$
\epsilon \dot{u}_{\mathrm{c}}(t)=-\alpha\left(f\left(\mathbf{x}\left(t-T_{d}\right), \mathbf{z}\left(t-T_{d}\right), u_{\mathrm{c}}(t)\right)+h(t)\right) .
$$

In contrast, the PI controller can be shown to be equivalent to

$\epsilon \dot{u}_{\mathrm{c}}(t)=-\alpha\left(f\left(\mathbf{x}\left(t-T_{d}\right), \mathbf{z}\left(t-T_{d}\right), u_{\mathrm{c}}\left(t-T_{d}\right)\right)+h(t)\right)$,

the difference being that $u_{\mathrm{c}}(t)$ enters the nonlinear function $f$ delayed by $T_{d}$.

\section{LINEAR TIME-INVARIANT SYSTEMS}

This section uses well established linear system techniques to compare some robustness properties of the closed loop system controlled by the PI controller (8) and by the ADI control law (6) when the system is minimum-phase and LTI. 
Consider the class of $\rho$-th order single-input minimumphase LTI systems described by

$$
\dot{\mathbf{x}}(t)=\mathbf{A} \mathbf{x}(t)+b \mathbf{c} u(t),
$$

where $b$ is a constant scalar satisfying $|b| \geq b_{0}>0$, and

$$
\mathbf{A}=\left[\begin{array}{cccc}
0 & 1 & \cdots & 0 \\
\vdots & \vdots & \ddots & \vdots \\
0 & 0 & \cdots & 1 \\
a_{0} & a_{1} & \cdots & a_{(\rho-1)}
\end{array}\right], \quad \mathbf{c}=\left[\begin{array}{c}
0 \\
\vdots \\
0 \\
1
\end{array}\right]
$$

\section{A. Input Sensitivity Function}

First, we show that the closed loop system controlled by the PI controller (8) has a superior input sensitivity function compared to that controlled by the ADI control law (6). The PI controller (8) applied to this system can be written as

$$
\epsilon \dot{u}_{\mathrm{PI}}(t)=-\operatorname{sign}(b) \mathbf{c}^{\mathrm{T}}\left(\dot{\mathbf{x}}(t)-\dot{\mathbf{x}}_{\mathrm{r}}(t)-\mathbf{A}_{\mathrm{e}}\left(\mathbf{x}(t)-\mathbf{x}_{\mathrm{r}}(t)\right)\right),
$$

where $\mathbf{A}_{\mathrm{e}}$ defines the error dynamics (3) and $\mathbf{x}_{\mathrm{r}}(t)$ is the state of the reference model (2).

Taking Laplace transforms of (18) and (19) yields

$$
\begin{aligned}
\mathbf{x}(s) & =b(s \mathbf{I}-\mathbf{A})^{-1} \mathbf{c} u(s), \\
u_{\mathrm{PI}}(s) & =-\frac{\operatorname{sign}(b)}{\epsilon s} \mathbf{c}^{\mathrm{T}}\left(s \mathbf{I}-\mathbf{A}_{\mathrm{e}}\right)\left(\mathbf{x}(s)-\mathbf{x}_{\mathrm{r}}(s)\right),
\end{aligned}
$$

respectively. Breaking the loop at the input to the system, the input loop transfer function is then

$$
L_{\mathrm{PI}}(s)=\frac{|b|}{\epsilon s} \mathbf{c}^{\mathrm{T}}\left(s \mathbf{I}-\mathbf{A}_{\mathrm{e}}\right)(s \mathbf{I}-\mathbf{A})^{-1} \mathbf{c} .
$$

The ADI controller (6) applied to the same system is

$$
\begin{aligned}
\epsilon \dot{u}_{\mathrm{ADI}}(t)= & -\operatorname{sign}(b) \mathbf{c}^{\mathrm{T}}\left(\mathbf{A} \mathbf{x}(t)+b \mathbf{c} u_{\mathrm{ADI}}(t)\right. \\
& \left.-\mathbf{A}_{\mathrm{r}} \mathbf{x}_{\mathrm{r}}(t)-\mathbf{B}_{\mathrm{r}} r(t)-\mathbf{A}_{\mathrm{e}}\left(\mathbf{x}(t)-\mathbf{x}_{\mathrm{r}}(t)\right)\right),
\end{aligned}
$$

with Laplace transform

$$
\begin{aligned}
u_{\mathrm{ADI}}(s)=-\frac{\operatorname{sign}(b)}{\epsilon s+|b|} \mathbf{c}^{\mathrm{T}}\left(\left(\mathbf{A}-\mathbf{A}_{\mathrm{e}}\right) \mathbf{x}(s)\right. \\
\left.+\left(\mathbf{A}_{\mathrm{e}}-\mathbf{A}_{\mathrm{r}}\right) \mathbf{x}_{\mathrm{r}}(s)-\mathbf{B}_{\mathrm{r}} r(s)\right) .
\end{aligned}
$$

The input loop transfer function is then

$$
L_{\mathrm{ADI}}(s)=\frac{|b|}{\epsilon s+|b|} \mathbf{c}^{\mathrm{T}}\left(\mathbf{A}-\mathbf{A}_{\mathrm{e}}\right)(s \mathbf{I}-\mathbf{A})^{-1} \mathbf{c} .
$$

The corresponding input sensitivity functions are

$$
S_{\mathrm{PI}}(s)=\left(1+L_{\mathrm{PI}}(s)\right)^{-1}, \quad S_{\mathrm{ADI}}(s)=\left(1+L_{\mathrm{ADI}}(s)\right)^{-1} .
$$

The following establishes a key relationship between the input sensitivity functions of identical systems controlled by the ADI and PI controllers.

Proposition 2: For any $\epsilon \in(0, \infty)$,

$$
S_{\mathrm{PI}}(s)=\frac{\epsilon s}{\epsilon s+|b|} S_{\mathrm{ADI}}(s) .
$$

For any $\epsilon \in\left(0, \epsilon_{*}\right)$, where $\epsilon_{*}$ is defined in Theorem 1 ,

$$
\left\|S_{\mathrm{PI}}(s)\right\|_{\infty}<\left\|S_{\mathrm{ADI}}(s)\right\|_{\infty} \text {. }
$$

Proof: From (20), we have

$$
1+L_{\mathrm{PI}}(s)=\frac{\epsilon s+|b| \mathbf{c}^{\mathrm{T}}\left(s \mathbf{I}-\mathbf{A}_{\mathrm{e}}\right)(s \mathbf{I}-\mathbf{A})^{-1} \mathbf{c}}{\epsilon s},
$$

and from (21),

$$
\begin{aligned}
1+L_{\mathrm{ADI}}(s) & =\frac{\epsilon s+|b|+|b| \mathbf{c}^{\mathrm{T}}\left(\mathbf{A}-\mathbf{A}_{\mathrm{e}}\right)(s \mathbf{I}-\mathbf{A})^{-1} \mathbf{c}}{\epsilon s+|b|} \\
& =\frac{\epsilon s+|b| \mathbf{c}^{\mathrm{T}}\left(\mathbf{I}+\left(\mathbf{A}-\mathbf{A}_{\mathrm{e}}\right)(s \mathbf{I}-\mathbf{A})^{-1}\right) \mathbf{c}}{\epsilon s+|b|} .
\end{aligned}
$$

Factoring out $(s \mathbf{I}-\mathbf{A})^{-1}$ on the right and canceling terms, yields

$$
\begin{aligned}
1+L_{\mathrm{ADI}}(s) & =\frac{\epsilon s+|b| \mathbf{c}^{\mathrm{T}}\left(s \mathbf{I}-\mathbf{A}_{\mathrm{e}}\right)(s \mathbf{I}-\mathbf{A})^{-1} \mathbf{c}}{\epsilon s+|b|} \\
& =\frac{\epsilon s}{\epsilon s+|b|}\left(1+L_{\mathrm{PI}}(s)\right),
\end{aligned}
$$

which proves (22) for any $\epsilon \in(0, \infty)$.

Next, observe from Theorem 1 that any choice of $\epsilon \in\left(0, \epsilon_{*}\right)$ results in a stable closed loop system. Then $\left\|S_{\mathrm{PI}}(s)\right\|_{\infty}$ and $\left\|S_{\mathrm{ADI}}(s)\right\|_{\infty}$ are both finite. Let $\left|S_{\mathrm{PI}}(j \omega)\right|$ attain its maximum at $\omega_{0}$ so that $\left\|S_{\mathrm{PI}}(s)\right\|_{\infty}=\left|S_{\mathrm{PI}}\left(j \omega_{0}\right)\right|$. From (22), at frequency $\omega_{0}$, we have

$$
\left|S_{\mathrm{ADI}}\left(j \omega_{0}\right)\right|=\left|1-j \frac{|b|}{\epsilon \omega_{0}}\right|\left|S_{\mathrm{PI}}\left(j \omega_{0}\right)\right|>\left\|S_{\mathrm{PI}}(s)\right\|_{\infty} .
$$

Since $\left\|S_{\mathrm{ADI}}(s)\right\|_{\infty} \geq\left|S_{\mathrm{ADI}}\left(j \omega_{0}\right)\right|,(23)$ is proved.

Observe that $\|S(s)\|_{\infty}$ is one measure of robustness, the reciprocal of which is the shortest Euclidean distance in the complex plane of the Nyquist plot from the critical point, $-1+j 0$. From (23), we see that the shortest distance between the Nyquist plot of $L_{\mathrm{PI}}(s)$ and the critical point is always larger than that of $L_{\mathrm{ADI}}(s)$. Hence the PI controlled system can tolerate larger perturbations to $L_{\mathrm{PI}}(s)$ while maintaining stability compared to the ADI controlled system. This shows that in terms of the input sensitivity function, the PI controller has better robustness properties.

\section{B. Time Delay Margin}

The time delay margin for a system with loop transfer function $L(s)$ is defined in [13] as

$$
T M_{\mathrm{sc}}=\frac{\text { phase margin }}{\text { gain crossover frequency }}=\frac{\phi_{\mathrm{m}}}{\left|\omega_{\mathrm{c}}\right|},
$$

where $\phi_{\mathrm{m}}$ and $\omega_{\mathrm{c}}$ are the phase margin and gain crossover frequency of $L(s)$ respectively. It is a measure of the amount of time delay that an LTI system can tolerate, beyond which the closed loop system loses stability. It is another measure of system robustness of practical importance. To be applicable to systems whose loop transfer functions have no, multiple or infinite number of crossovers, we use the modified definition

$$
\begin{array}{r}
T M=\inf \left\{\phi_{\mathrm{m}} /\left|\omega_{\mathrm{c}}\right| \in \mathbb{R}\left|\exists \omega_{\mathrm{c}} \in \mathbb{R},\right| L\left(j \omega_{\mathrm{c}}\right) \mid=1,\right. \\
\left.\phi_{\mathrm{m}}=\left(\left(\angle L\left(j \omega_{\mathrm{c}}\right) \bmod 2 \pi\right)-\pi\right)\right\},
\end{array}
$$

with the convention that the infimum of an empty set is $+\infty$. Note that implicit in the above definition is that $\phi_{\mathrm{m}} \in$ $[-\pi, \pi)$. 
Here, we show that as $\epsilon \rightarrow 0$, the time delay margin of the system controlled by the PI equivalent, $T M_{\mathrm{PI}}$, approaches zero. This means that if there are any (non-zero) finite time delays present within the loop, the system can be destabilized if $\epsilon$ is made too small.

Proposition 3: The time delay margin of the closed loop system stabilized by the PI controller (8) satisfy

$$
\lim _{\epsilon \rightarrow 0} T M_{\mathrm{PI}}=0 \text {. }
$$

Proof: Since the closed loop system is stable by assumption, the phase margin satisfy $\phi_{\mathrm{m}} \in[0, \pi)$ if there exists at least one real $\omega_{c}$ such that $\left|L_{\mathrm{PI}}\left(j \omega_{c}\right)\right|=1$, ie., that there is at least one gain crossover point. Hence it is sufficient to show that when $\epsilon \rightarrow 0$, there exists a solution $\omega_{c} \in \mathbb{R}$ satisfying $\left|L_{\mathrm{PI}}\left(j \omega_{c}\right)\right|=1$ such that $\left|\omega_{c}\right| \rightarrow \infty$.

From (20), it can be shown that $L_{\mathrm{PI}}(s)$ expands to

$$
\begin{aligned}
L_{\mathrm{PI}}(s) & =\frac{|b|}{\epsilon s} \frac{s^{\rho}+a_{\mathrm{e}(\rho-1)} s^{\rho-1}+\cdots+a_{\mathrm{e} 1} s+a_{\mathrm{e} 0}}{s^{\rho}-a_{(\rho-1)} s^{\rho-1}-\cdots-a_{1} s-a_{0}}, \\
& :=\frac{|b|}{\epsilon s} \frac{p(s)}{q(s)} .
\end{aligned}
$$

It can be seen that since $L_{\mathrm{PI}}(s)$ is strictly proper, and has a pole at $s=0$, we have that

$$
\lim _{\omega \rightarrow \infty}\left|L_{\mathrm{PI}}(j \omega)\right|=0, \quad \quad \lim _{\omega \rightarrow 0}\left|L_{\mathrm{PI}}(j \omega)\right|=\infty .
$$

By the continuity of $\left|L_{\mathrm{PI}}(j \omega)\right|$ with $\omega$, there must exist a real $\omega_{c}$ that satisfy

$$
\left|L_{\mathrm{PI}}\left(j \omega_{c}\right)\right|=\frac{|b|}{\epsilon\left|\omega_{c}\right|} \frac{\left|p\left(j \omega_{c}\right)\right|}{\left|q\left(j \omega_{c}\right)\right|}=1 .
$$

Rearranging terms and taking limits, we have

$$
\lim _{\epsilon \rightarrow 0} \epsilon=0=\frac{|b|}{\left|\omega_{c}\right|} \frac{\left|p\left(j \omega_{c}\right)\right|}{\left|q\left(j \omega_{c}\right)\right|} .
$$

Observe that $p(s)$ is the characteristic polynomial of $\mathbf{A}_{\mathrm{e}}$. Since $\mathbf{A}_{\mathrm{e}}$ is Hurwitz, all roots of $p(s)=0$ have negative real parts, so that none lies on the $j \omega$ axis and $\forall \omega \in \mathbb{R}$, $|p(j \omega)| \neq 0$. Since $p(s)$ and $q(s)$ are of the same order, we must have from (24) that $\left|\omega_{c}\right| \rightarrow \infty$.

This shows that there is a practical lower bound of $\epsilon$ when implementing the equivalent PI controller. In contrast, for the limiting case $\epsilon \rightarrow 0$, we see from (21) that $\lim _{\epsilon \rightarrow 0} T M_{\mathrm{ADI}}$ is defined entirely by

$$
\lim _{\epsilon \rightarrow 0} L_{\mathrm{ADI}}(s)=\mathbf{c}^{\mathrm{T}}\left(\mathbf{A}-\mathbf{A}_{\mathrm{e}}\right)(s \mathbf{I}-\mathbf{A})^{-1} \mathbf{c},
$$

and does not exhibit the zero delay tolerance characteristic of the PI controlled system.

\section{Conclusions}

An extension of the Approximate Dynamic Inversion (ADI) method for minimum-phase nonaffine-in-control systems was presented that renders the error dynamics independent of the reference model dynamics. In essence, this decouples the "steady state" response specification from the transient response specification, where the "steady state" response is specified by the reference model dynamics while the transient response is independently specified by the error dynamics. It was shown that every ADI control law admits an equivalent linear Proportional-Integral (PI) controller realization that is largely independent of the nonlinearities of the system.

This equivalence holds only for the time response, and only when applied to the exact system. In particular, even when specializing to minimum-phase linear time-invariant systems, they differ in robustness properties. In terms of the input sensitivity function, the PI controller was shown to be more robust than the ADI controller. In terms of time-delay margin, the ADI controller is superior.

For the practitioner, the choice between implementing the original ADI control law or PI equivalent lies in whether a sufficiently accurate characterization of the system is available, or whether time delays are the major limiting factor in the system. An interesting research topic not specifically linked to the ADI or PI controller is, among all equivalent controllers, to find those with superior robustness properties.

\section{ACKNOWLEDGMENTS}

The first author gratefully acknowledges the support of DSO National Laboratories, Singapore. Research funded in part by AFOSR grant FA9550-08-1-0086.

\section{REFERENCES}

[1] S. Devasia, D. Chen, and B. Paden, "Nonlinear inversion-based output tracking," IEEE Trans. Autom. Control, vol. 41, no. 7, pp. 930 - 942, Jul. 1996.

[2] H. K. Khalil, Nonlinear Systems, 3rd ed. Upper Saddle River, NJ: Prentice Hall, 2002.

[3] G. O. Guardabassi and S. M. Savaresi, "Approximate linearization via feedback - an overview," Automatica, vol. 37, no. 1, pp. 1 - 15, Jan. 2001.

[4] N. Hovakimyan, E. Lavretsky, and A. Sasane, "Dynamic inversion for nonaffine-in-control systems via time-scale separation. part I," J. Dyn. Control Syst., vol. 13, no. 4, pp. 451 - 465, Oct. 2007.

[5] A. Chakrabortty and M. Arcak, "Time-scale separation redesigns for stabilization and performance recovery of uncertain nonlinear systems," Automatica, vol. 45, no. 1, pp. 34 - 44, Jan. 2009.

[6] J. Teo and J. P. How, "Equivalence between approximate dynamic inversion and proportional-integral control," in Proc. 47th IEEE Conf. Decision and Control, Cancun, Mexico, Dec. 2008, pp. 2179 - 2183.

[7] P. V. Kokotovic and R. Marino, "On vanishing stability regions in nonlinear systems with high-gain feedback," IEEE Trans. Autom. Control, vol. AC-31, no. 10, pp. 967 - 970, Oct. 1986.

[8] H. K. Khalil, "Universal integral controllers for minimum-phase nonlinear systems," IEEE Trans. Autom. Control, vol. 45, no. 3, pp. 490 - 494, Mar. 2000.

[9] J. Teo and J. P. How, "Equivalence between approximate dynamic inversion and proportional-integral control," MIT, Cambridge, MA, Tech. Rep. ACL08-01, Sep. 2008, Aerosp. Controls Lab. [Online]. Available: http://hdl.handle.net/1721.1/42839

[10] J. P. How, J. Teo, and B. Michini, "Adaptive flight control experiments using RAVEN," in Proc. 14th Yale Workshop Adaptive and Learning Systems, New Haven, CT, Jun. 2008, pp. $205-210$.

[11] N. Hovakimyan, E. Lavretsky, and C. Cao, "Adaptive dynamic inversion via time-scale separation," in Proc. 45th IEEE Conf. Decision and Control, San Diego, CA, Dec. 2006, pp. 1075 - 1080.

[12] E. Lavretsky and N. Hovakimyan, "Adaptive compensation of control dependent modeling uncertainties using time-scale separation," in Proc. 44th IEEE Conf. Decision and Control \& European Control Conf., Seville, Spain, Dec. 2005, pp. 2230 - 2235.

[13] C. Cao, V. V. Patel, C. K. Reddy, N. Hovakimyan, E. Lavretsky, and $\mathrm{K}$. Wise, "Are phase and time-delay margins always adversely affected by high-gain?" in Proc. AIAA Guidance Navigation and Control Conf. and Exhibit, Keystone, CO, Aug. 2006, AIAA-2006-6347. 